\title{
Electrochemical studies of silver nanoparticles: a guide for experimentalists and a perspective
}

\author{
Kristina Tschulik, Christopher Batchelor-McAuley, Her-Shuang Toh, Emma J E Stuart, and Richard G \\ Compton
}

\author{
Received (in $X X X, X X X)$ Xth $X X X X X X X X X 20 X X$, Accepted $X$ th $X X X X X X X X X 20 X X$ \\ DOI: $10.1039 / b 00000 x$
}

This perspective summarises four different electrochemical techniques that have been established and frequently used to characterize various properties of silver nanoparticles. These are based on drop casting

10 (I), in situ nanoparticle sticking and stripping (II), transfer sticking and stripping (III) or nanoparticle impacts (IV). The specific characteristics of the different methodologies are explained and contrasted with each other with the focus being on the respective benefits and limitations together with essential insights for experimentalists.

The trend towards multi-functional materials and devices today 15 not only applies for electrical gadgets such as smart phones, but also affects almost every type of consumer product. ${ }^{1}$ One way producers seek to satisfy demand is to modify standard products with nanoparticles; silver nanoparticles are amongst the most prominent examples for this. The textile industry offers fast20 drying fashionable sport shirts and socks and the addition of silver nanoparticles to these textiles moreover reduces unwanted odours. ${ }^{1.2}$ Medical implants, plasters and refrigerators impregnated with silver nanoparticles are just as commonly available as deodorants containing silver nanoparticles. ${ }^{2}$ By 25 introducing silver these products gain the functionality of being 'antibacterial' or more generally 'antimicrobial'. The extra production costs are kept at a minimum by using nanoparticulate silver, that is only a small amount of the relatively expensive noble metal. The increased use of these nanoparticles goes hand 30 in hand with their increased release into the environment. Yet, neither their ways of distribution into, nor the impact of their 'antimicrobial' action on eco - and biological systems are fully understood ${ }^{3,4}$. The enormous variety of environmental matrices, their often heterogeneous character and the low concentration of 35 nanoparticles, render the development of reliable, simple and fast detection and characterization techniques one of today's most challenging analytical tasks. ${ }^{5}$ Since additionally nanoparticles are known to transform quickly when changing their direct environment (e.g. $\mathrm{pH}$, ion concentration or surface active 40 molecules in solution), a lot of effort has been made in recent years to develop suitable analytical techniques for a broad spectrum of analytical matrices. ${ }^{1,6-8}$

Electrochemical techniques represent a particularly useful approach towards solving these issues, as in contrast to many 45 other techniques they allow for both analysis in the liquid phase and in presence of high levels of salt. For environmental samples these electrochemical approaches have been proven to be very useful due to their ability to detect, to size and to quantify silver nanoparticles, ${ }^{9-11}$ even in highly complex matrices such as sea so water, which intrinsically contains high concentrations of sodium chloride and other salts. In this field several methodologies have been employed, depending on the respective research focus. Sizing of individual silver nanoparticles by measuring the oxidative charge during their quantitative anodic dissolution upon 55 impacting on a micro electrode has been demonstrated for laboratory-made silver nanoparticles and well-defined electrolytes. ${ }^{12}$ Recently it has been shown that the same method can be applied for the detection of commercial silver nanoparticles in real sea water samples. ${ }^{13}$

${ }_{60}$ Besides this analytical demand, studying silver nanoparticles is also highly interesting from a fundamental point of view. Starting from a metallic bulk material, such as silver, the successive reduction of the particle characteristic length scale down to the low nanometer region causes drastic changes in both the physical 65 and electronic structure. For metallic particles with a diameter of less than $1 \mathrm{~nm}$, the confinement of the electrons present in the material significantly alters the electronic structure. ${ }^{14,15}$ Even prior to the strong confinement limit, much larger nanoparticles may exhibit distinctly different chemical behaviour from the 70 corresponding bulk material; an observation that is less well understood than the classic quantum size effect, but could reflect altered surface topography. Considering that for a nanoparticle of $10 \mathrm{~nm}$ diameter half of the nanoparticle atoms are on its surface, ${ }^{14}$ nanoparticle properties of this larger size are mostly governed by 75 the high ratio of surface to bulk atoms. Consequently, significant changes in the thermodynamics and kinetics of reactions involving silver nanoparticles instead of macroscopic silver can be anticipated. Bearing in mind the variety of electrochemical processes involving silver as a catalyst or as the reactive species, 


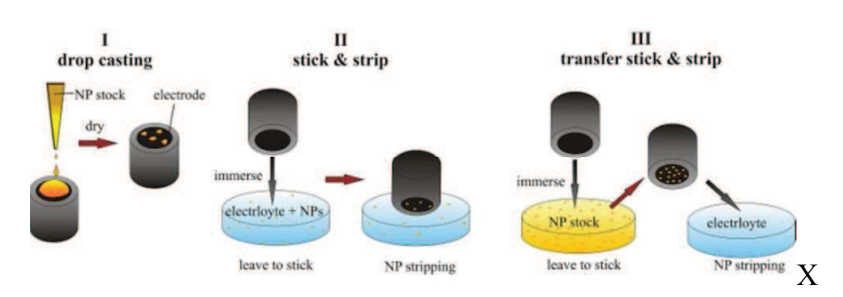

Scheme 1 Common approaches to nanoparticle modification of electrodes.

5 a wide field of fundamental issues is to be addressed. ${ }^{16}$

Beyond the consideration of the inherent properties of the nanoparticulate material itself, the mass-transport to and from the surface may also be influential in altering the observed reactivity of the material on the sub-micrometer scale. As an example, the

10 higher toxicity of silver nanoparticles as compared to bulk silver, which is still under debate, ${ }^{4,17}$ has recently been explained in a rigorous theoretical argumentation. ${ }^{18}$ Considering the reaction kinetics of electrochemical and chemical reactions involved in typical cellular environments, (i.e. oxygenated aqueous media 15 with high concentrations of salts and near-neutral $\mathrm{pH}$ ) silver nanoparticles are found not only to readily aerially oxidize, with the coupled formation of $\mathrm{Ag}^{+}$ions, but an accompanied release of hydrogen peroxide is predicted, which is not the case for larger (multi micrometer-sized) silver particles. This alteration of the 20 mechanism is a result of the higher rates of diffusional flux of the intermediate oxygen reduction products (essentially $\mathrm{H}_{2} \mathrm{O}_{2}$ ) away from the metal-electrolyte interface at nanoparticles as compared to bulk silver. Furthermore, hydrogen peroxide is well-known to cause additional oxidative stress on living cells and thus its

25 formation during silver nanoparticle oxidation in a biological sample can explain the observed toxicity increase with respect to bulk silver.

Despite their ability to yield significant and reliable information electrochemical experiments regarding the behaviour of silver 30 nanoparticles in various liquid environments, both for fundamental investigations and for analytical purposes, have to be set up and performed in an appropriate way to avoid misinterpretation of data. While researchers today are aware that, for example, the increased surface area and altered mass transport 35 can easily be mistaken for an enhanced catalytic activity of nanoparticles with respect to their bulk counter parts, a variety of other sources of misinterpretation might come into play. In this 'perspective' we aim to present a number of hidden traps and pitfalls to be avoided during electrochemical studies involving

40 silver nanoparticles. In particular the effects of drop casting (I) as compared to the solution based modification during in situ nanoparticle sticking and stripping (II) or transfer sticking and stripping (III), as well as nanoparticle impact (IV) experiments will be addressed. The various parameters affecting the solution

45 based sticking, such as the used electrolyte, an applied standby potential or the chemical nature of the working electrode, will be addressed and from these observations suggestions will be derived on which electrochemical methodology appears most appropriate for either analytical or fundamental investigations.

\section{Drop casting techniques}

'Drop casting', as the name implies, denotes the process of ${ }_{55}$ casting a drop of a suspension or solution on an electrode surface with subsequent evaporation of the solvent to immobilise a species of interest on a surface. In nano-electrochemistry drop casting is frequently used to modify macro electrodes with nanoparticles, which then, for instance, can be used to determine 60 suitable potentials for reduction or oxidation of these nanoparticles. However, as with bulk silver and all electrochemical nanoparticle investigations, the role of the supporting electrolyte used should be considered. Specifically, in the case of silver the strong complexation of the $\mathrm{Ag}^{+}$ions to,

${ }_{65}$ among others, halide anions must be taken into account, even at sub-micro molar level. In fact, it is this sensitivity of the silver oxidation process to the electrolyte that has allowed drop casting of silver nanoparticles on a carbon surface to be utilised for the quantitative detection of chloride ions in solution. ${ }^{19}$ In this 70 example, the finite amount of silver available on the electrode surface allows for detection of either a single oxidation peak (corresponding to the formation of $\mathrm{Ag}^{+}$ions) or, in the presence of chloride ions in the solution, of two oxidation peaks. Thereby, the additional peak (corresponding to silver chloride formation) is 75 detected at lower anodic potentials and its peak current increases linearly with increasing concentration of chloride in the sample solution over a wide range. A proof-of-concept sensor utilising this effect proved quantitative detection of typical chloride levels in artificial sweat, thus demonstrating the potential use of silver 80 nanoparticle modified electrodes for fast detection of cystic fibrosis, a common disease indicated by a high chloride content in patients' sweat. ${ }^{19}$

Alternatively, if a suitable non-complexing and non-ion-pairing electrolyte is used then fundamental studies of the silver 85 nanoparticles and their associated energies may be facilitated. Note the presence of silver-complexing anions not only alters the formal potential for the oxidation process but the possible formation of nanoparticulate silver complexes, such as $\mathrm{AgCl}$, makes the quantitative analysis of the voltammetric data 90 challenging. Conversely, if the oxidative product is a solution phase $\mathrm{Ag}^{+}$ion the analysis is partially simplified. However, here the mass-transport of the formed ions away from the metallic surface must be accounted for. In a recent work of Toh et al. ${ }^{20}$ a general analytical description of the voltammetric stripping 95 behaviour of nanoparticle dissolution, as a function of the surface coverage of the electrode, was developed. For high surface coverage the diffusion layers of the formed $\mathrm{Ag}^{+}$of individual nanoparticles strongly overlap, under these conditions a linear diffusion regime (so called case $4^{21}$ ) to the electrode surface is 100 established, and an increase of the anodic peak potential with increasing surface coverage is predicted. In contrast, for very low nanoparticle coverage the diffusion layers of neighbouring nanoparticles do not overlap (case 2) and the mass transport to each of the diffusionally-independent nanoparticles is governed 105 by radial diffusion, which is dependent upon the size of the nanoparticle, but not on the surface coverage. Note that 'low' and 'high' coverages relate to whether the average nanoparticle separation is large or small as compared to the distance $\mathrm{Ag}^{+}$ions diffuse on the time scale of the experiments, which is $(2 D t)^{0.5}$ 110 where $D$ is the diffusion coefficient and $t$ the time. 


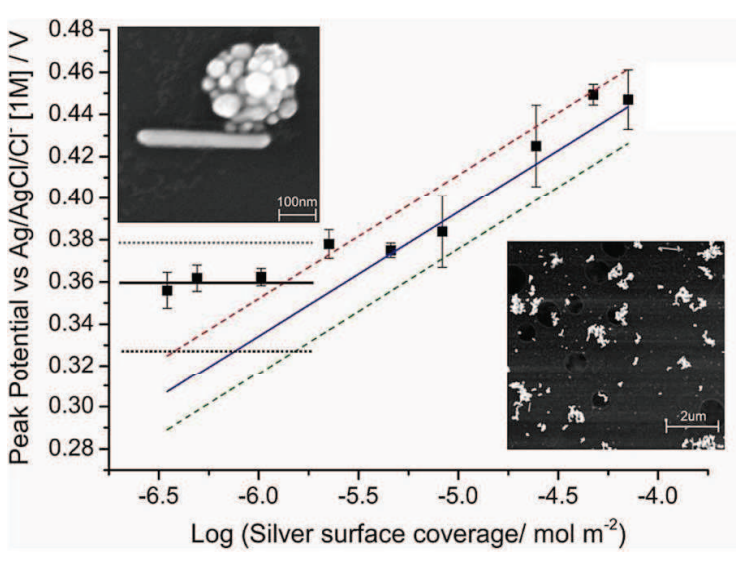

Fig. 1 Measured dependence of the oxidation potential for silver nanoparticle stripping from a macro glassy carbon electrode as a function of surface coverage (black squares). For high surface coverage the stripping potential increases linearly as predicted theoretically (reversible case 4 , coloured lines). The constant stripping potential at lower surface coverage (reversible case 2, grey-scaled lines) allows for sizing of the surface immobilised silver nanoparticles. Theoretical values for different radii are depicted by grey dots $(100 \mathrm{~nm})$, black line $(80 \mathrm{~nm})$ and black

10 dots $(40 \mathrm{~nm})$. Both, electrochemical sizing and SEM images (inlays) show particle sizes of $80 \mathrm{~nm}$ radius, which results from agglomeration of the originally $13 \mathrm{~nm}$ sized nanoparticles upon drop casting onto a surface. (images taken and reassembled from ${ }^{20}$ ).

15 At a superficial level this drop casting technique upon a macro electrode may appear ideal for fundamental studies. However, the afore-mentioned sensitivity of nanoparticles to their direct environment can play a major role during drop casting experiments. During evaporation of the solvent, destabilization 20 and hence agglomeration or aggregation ${ }^{1}$ of formerly single nanoparticles may occur. To experimentally verify this hypothesis, the drop casting method was applied as it provides a route for direct control of the surface coverage by adjusting the volume of silver nanoparticle suspension drop cast onto an 25 electrode surface. As depicted in Figure 1 for different amounts of drop cast silver, the authors observed the predicted linear dependency of the silver stripping potential for high surface coverage and a near constant stripping potential for low surface coverage. ${ }^{20}$ Yet, the nanoparticle size calculated from the (almost 30 constant) peak potential at low surface coverage, yielded a nanoparticle radius of $\sim 80 \mathrm{~nm}$. This was about six times bigger than the nanoparticle radius in solution, as had been determined by UV-vis spectroscopy and nanoparticle tracking analysis. Additional SEM characterization of a drop cast sample, however, 35 confirmed that indeed big agglomerates of $80 \mathrm{~nm}$ size were formed (see inlay in Fig. 1), confirming that electrochemical means can be used to precisely analyse the size of silver nanoparticle 'clusters' immobilised onto an electrode. Hence, in this setup the electrochemically interrogated nanoparticle 'size' 40 does not necessarily represent that of the individual nanoparticles as found in suspension.

As shall be discussed in the next section, for fundamental electrochemical studies, such as to assess and quantify size dependent changes of thermodynamic data or reaction kinetics,

45 solution-based nanoparticle adsorption should be preferred over drop casting approaches. These solution based 'sticking' approaches include the immobilization of nanoparticles onto an electrode during its immersion into a nanoparticle suspension, hence reducing solvent evaporation related agglomeration.

\section{II in situ nanoparticle sticking and stripping}

In situ nanoparticle sticking and stripping experiments allow for adsorption of nanoparticles on an electrode surface via immersion into a nanoparticle containing electrolyte for variable amounts of 55 time. In this way nanoparticle agglomeration upon immobilization on a surface is minimised as compared to the afore-mentioned drop casting method. The adsorption is then followed by the subsequent oxidation of the adsorbed material. By analogy to nomenclature used within surface chemistry the 60 probability of an impacting nanoparticle to adhere to the electrode may be defined by its sticking coefficient. ${ }^{23}$ For particles of 14 and $45 \mathrm{~nm}$ in radius the probability of the particle remaining on the surface after impact was found to be constant (for sticking onto a carbon micro electrode in $0.1 \mathrm{M}$ sodium ${ }_{65}$ perchlorate solution). Beyond the study of the adsorption process this 'stick and strip' electrode modification method has been utilised for the study of nanoparticle surface energies. ${ }^{24}$ Here, the in-situ adsorption based methodolgy is utilised to minimise surface agglomeration of the nanoparticles (as compared to the 70 drop casting methodology) and allows the controlled modification of a micro electrode. An experimentally observed shift in the voltammetric oxidation stripping potential is recorded for the relatively small ( $3.5 \mathrm{~nm}$ radius) and large (13.5 $\mathrm{nm}$ radius) silver nanoparticles. This shift in potential is quantitatively 75 explained by taking into account the increased surface energy of small nanoparticles, as predicted by Plieth in $1982 .{ }^{25}$

One drawback of this otherwise convenient approach of repetitive use of the same electrolyte for sticking and stripping of silver nanoparticles is that typically low nanoparticle concentrations are 80 added to the electrolyte so as to prevent major agglomeration of the nanoparticles in the presence of the relatively high ionic strength required for the electrochemical stripping step. ${ }^{2}$ Due to this low concentration and the fact that the double layer structure at the electrode and hence the potential of zero charge strongly 85 depend on the electrolyte used, nanoparticle sticking under open circuit conditions is often very time consuming and/or irreproducible. ${ }^{10}$ To increase the likelihood of silver nanoparticles adhering to the electrode, a standby potential is commonly applied to the electrode during the sticking step. For citrate90 capped silver nanoparticles a standby potential more negative than the open circuit potential has been found to be suitable. The sticking rate was reported to remain constant for further negative standby potentials. ${ }^{23}$

In the absence of solution phase $\mathrm{Ag}^{+}$ions, the applied standby ${ }_{95}$ potential will not influence the subsequent stripping results. However, in presence of $\mathrm{Ag}^{+}$ions the applied negative potential might not only facilitate the sticking of nanoparticles from the solution, but may also result in the electrochemical reduction of the $\mathrm{Ag}^{+}$ions. This problem is particularly significant for small 100 particles where even minimal deposition may alter the particle characteristics. $^{3}$ 


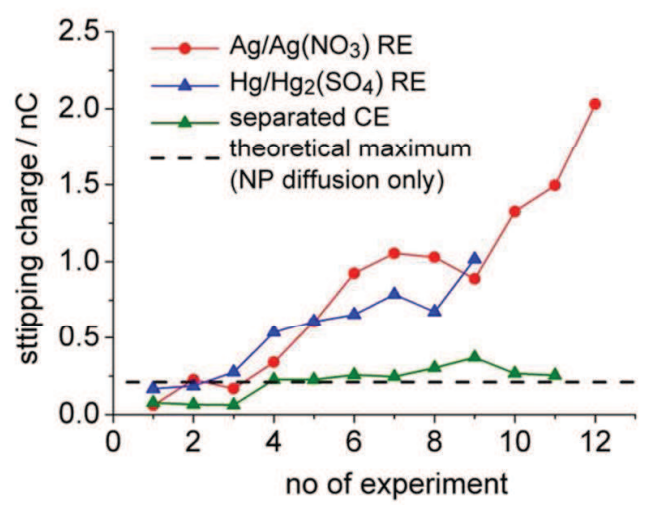

Fig. 2 Change of the silver stripping charge with increasing number of experiments using the same electrolyte. For each experiment a freshly polished carbon micro electrode was immersed into the electrolyte for 15 5 minutes, applying a negative holding potential and the adhered amount of silver was subsequently determined by linear stripping voltammetry; using a $\mathrm{Ag} / \mathrm{AgNO}_{3}$ reference electrode (red dots), or a mercury/mercury sulphate reference electrode in a one-compartment electrochemical cell (blue triangles) and a two-compartment cell separating the counter 10 electrode (platinum mesh) via a ceramic frit. The 'broken black line marks the theoretical maximum charge for diffusion-only silver nanoparticle sticking, assuming 100\% adsorption (unpublished data).

One major source of $\mathrm{Ag}^{+}$impurities is their intrinsic formation 15 within the electrochemical cell. When for example, several of these sticking and stripping experiments are performed using the same electrolyte, the detected silver stripping charge can increase significantly. To demonstrate this the following set of experiments is illustrative. A carbon fiber micro electrode $(\varnothing=$

$2011 \mu \mathrm{m}$ ) is immersed into a $0.01 \mathrm{M}$ solution of sodium nitrate containing $146 \mathrm{pM}$ of silver nanoparticles of $4 \mathrm{~nm}$ radius for 15 minutes, applying a standby potential of $-700 \mathrm{mV}$ vs $\mathrm{Ag} / \mathrm{Ag}\left(\mathrm{NO}_{3}\right)$ reference electrode ${ }^{4}$. The surface immobilised silver is subsequently oxidised by linear sweep voltammetry from -0.3

${ }_{25} \mathrm{~V}$ to $0.05 \mathrm{~V}$ vs a $\mathrm{Ag} / \mathrm{Ag}\left(\mathrm{NO}_{3}\right)$, yielding the respective silver stripping charge. After each stick and strip experiment, the working electrode is thoroughly polished to avoid any influence of possibly adhering material and the electrode is re-immersed into the electrolyte used for the previous experiment. Since the

30 amount of silver nanoparticles immobilised and dissolved during each experiment is small as compared to the total nanoparticle concentration in the electrolyte, a constant stripping charge could be expected for repeating experiments. The maximum stripping charge, this is the charge resulting from surface adsorption of 35 every nanoparticle hitting the electrode (sticking coefficient $=1$ ), can be calculated to be $0.21 \mathrm{nC}$ assuming steady-state convergent diffusion to the micro electrode.

As depicted in Figure 2, the detected silver stripping charge is not constant, but increases steadily with increasing number of 40 experiments, i.e. with repetitive use of the electrolyte (red dots). Notably, the amount of silver dissolved from the surface is much higher than the theoretical maximum for a diffusion-only process assuming $100 \%$ sticking of the silver nanoparticles. Since convection inside the electrolyte was carefully avoided, the 45 additional charge has to be attributed to immobilization of silver onto the surface through electrodeposition of $\mathrm{Ag}^{+}$during the immersion time. Thus, the used $\mathrm{Ag} / \mathrm{Ag}\left(\mathrm{NO}_{3}\right)$ reference electrode as one source of $\mathrm{Ag}^{+}$impurities is replaced by a mercury sulphate electrode $\left(\mathrm{Hg} / \mathrm{Hg}_{2}\left(\mathrm{SO}_{4}\right), \mathrm{K}_{2}\left(\mathrm{SO}_{4}\right)_{\text {sat }}\right)$ and a fresh electrolyte is ${ }_{50}$ used to repeat the previous experiments. As visible from the blue curve in Figure 2, the same trend as for the $\mathrm{Ag} / \mathrm{Ag}\left(\mathrm{NO}_{3}\right)$ reference electrode is observed, indicating that the $\mathrm{Ag}^{+}$impurities did not originate from reference electrode leakage.

An alternative source of $\mathrm{Ag}^{+}$ions is the counter electrode, where 55 in the course of the electrochemical experiments oxidation of silver nanoparticles can occur. Thus, another set of experiments was performed using a mercury sulphate reference electrode and separating the counter electrode ${ }^{5}$ from the other electrodes via a ceramic frit. So, a more moderate increase of the silver stripping 60 with an increasing number of experiments is observed, as depicted in Figure 2 (green triangles). The fact that even for this optimised setup an increasing stripping charge results, likely originates from the fact that $\mathrm{Ag}^{+}$ions formed at the counter electrode are not entirely separated from the remaining cell but ${ }_{65}$ some leakage of $\mathrm{Ag}^{+}$through the frit takes place.

Additionally, as it has been discussed in the literature very recently, ${ }^{18} \mathrm{Ag}^{+}$ions can be formed during the chemical oxidation of silver nanoparticles in the electrolyte in the presence of small amounts of oxygen, present even after purging with inert gas.

70 Depending on the applied method of nanoparticle synthesis and the storage conditions (freshly prepared, kept under inert gas atmosphere or under air), $\mathrm{Ag}^{+}$ions can already be present in the silver stock suspension, which upon its addition to an electrolyte will introduce $\mathrm{Ag}^{+}$impurities.

${ }_{75}$ Consequently, the repeated use of an electrolyte should be avoided due to the inherent risk of $\mathrm{Ag}^{+}$ion contamination of the electrolyte. Solution based silver nanoparticle sticking should ideally be performed under open circuit conditions. As discussed above, this is usually not very effective in electrolyte solutions 80 containing small amounts of nanoparticles. Thus, preferably the methodology of 'transfer sticking and stripping' should be employed. There, surface immobilization of nanoparticles is performed in a suspension of very low ionic strength, thus minimizing agglomeration, and the stripping is carried out 85 afterwards in a suitable electrolyte of high ionic strength.

\section{Transfer sticking and stripping}

This solution-based nanoparticle modification method involves the sticking of silver nanoparticles during immersionin a highly 90 concentrated suspension, such as the nanoparticle stock suspension, under open circuit conditions. The nanoparticle modified electrode is then transferred to an electrolyte where stripping of the adherent nanoparticles is performed. A first 


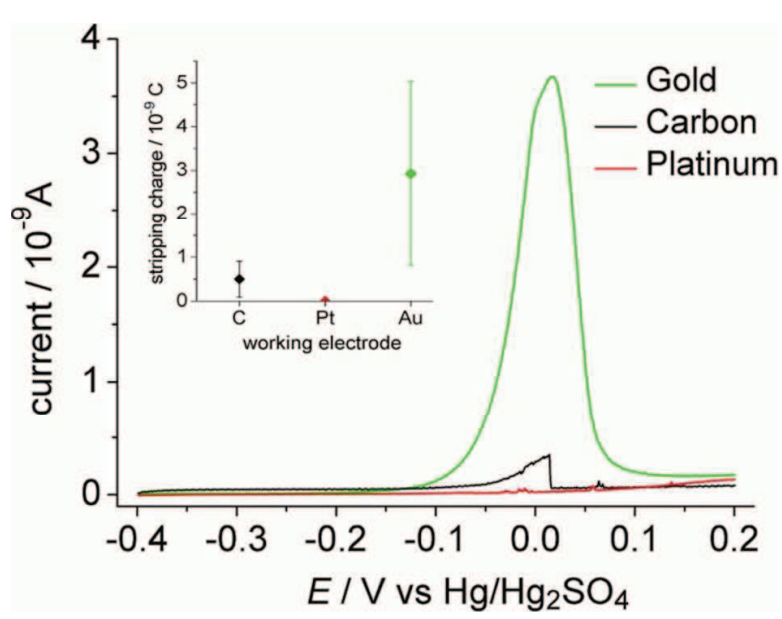

Fig. 3 Linear sweep stripping voltammograms showing the silver nanoparticle oxidation for three different micro electrodes: gold (green), carbon (black) and platinum (red). The electrodes were modified by

5 immersing them in a stock suspension of $4 \mathrm{~nm}$ sized silver nanoparticle for 930 minutes under open circuit conditions. After the transferring of the modified electrodes into a suitable electrolyte $(0.1 \mathrm{M}$ solution of trisodium citrate), oxidative linear stripping was performed from $-0.4 \mathrm{~V}$ to $0.2 \mathrm{~V}$ against a mercury/mercury sulphate electrode at a sweep rate of 50

$10 \mathrm{mV} / \mathrm{s}$. The average stripping charge for each electrode material detected over three repeats is plotted in the inlay (unpublished data).

advantage of this methodology is that a very low ionic strength of the sticking electrolyte can be maintained. This way 15 agglomeration of the nanoparticles in the solution is widely avoided and silver nanoparticles remain stable even at higher nanoparticle concentrations. A second advantage is that a wide range of electrolytes can be utilised for the stripping step, so as to perform fundamental studies even in extremely acidic or alkaline 20 solution, which would cause fast agglomeration of silver nanoparticles in solution and thus render in-situ sticking and stripping impossible.

One limitation of this method however is that silver nanoparticle sticking under open circuit conditions can depend on the working

25 electrode material used. To demonstrate this, transfer sticking experiments using a stock suspension of citrate-capped silver nanoparticles (radius $=4 \mathrm{~nm}$ ) are performed, immersing a carbon fiber-, a platinum- and a gold micro electrode into the suspension for 930 minutes. Afterwards, each electrode is transferred into a 30 stripping solution and the amount of adhering silver is determined by anodic linear sweep voltammetry. The stripping charges obtained for the three different electrodes differ significantly, as can be seen in Figure 3.

While for experiments using a platinum micro electrode no 35 oxidative peak is observed, a nearly symmetrical stripping peak is detected for a gold micro electrode. Linear sweep voltammograms, recorded using a carbon micro electrode, typically show an abrupt decay of the oxidative signal. The relatively large error bars included in the inlay of Fig. 3, 40 demonstrate the rather wide variability of the amount of nanoparticles immobilised on these surfaces.

A simple way to overcome the afore-mentioned problems of transfer sticking \& stripping experiments is to use electrodes with increased affinity for silver, increasing the silver nanoparticle 45 sticking coefficient and reducing the risk of nanoparticle drop-off

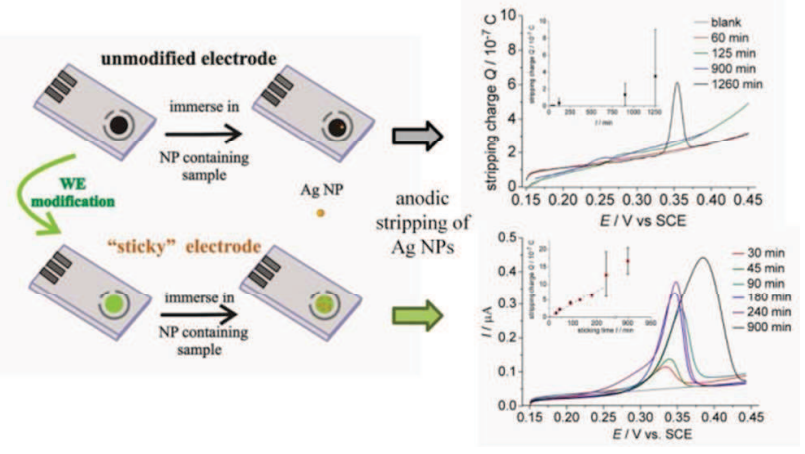

Fig. 4 Schematic drawing illustrating the pre-modification process of an electrode to increase the surface affinity for silver nanoparticle sticking 50 under open circuit conditions; exemplary silver nanoparticle stripping curves and charges (inlay) for an unmodified (top) and a cysteinemodified glassy carbon surface (b) for various immersion times in nanoparticle suspensions are shown on the right side of the image (partially adapted from ${ }^{10}$ ).

during the transfer stripping experiment. This approach is schematically summarised in Fig. 4, for the modification of a carbon electrode. It has been demonstrated that, for example, this enables the immobilisation of silver nanoparticles from 60 environmental seawater samples with reduced immersion time and better reproducibility ${ }^{10}$. The subsequent quantification by oxidative stripping can be performed in an electrolyte of choice. While the use of pre-modified 'sticky' electrodes can be extremely beneficial for analytical purposes, that is for detection 65 and quantification of silver nanoparticles in a sample of interest, the unknown - and likely strong - interactions between the surface-modifying compound (e.g. cysteine) and the silver nanoparticle make this method less suitable for fundamental studies, such as quantification of size-depended changes in 70 surface energy and oxidation potential, which among other previously mentioned issues is a question of major interest. Therefore, nanoparticle impact experiments remain as the suggested method of choice when addressing fundamental electrochemical questions.

\section{Impact experiments}

One important benefit of nanoparticle impact experiments is that individual nanoparticles can be analysed directly upon their impact onto a micro electrode. This is achieved by applying a 80 suitable potential to an inert micro electrode immersed in an electrolyte that contains nanoparticles. The Brownian motion of the nanoparticles causes nanoparticle-electrode collisions, and only during these collision the nanoparticle attains this applied potential and hence acts as an electrode. Thus, either mediated 85 reactions, such as hydrogen ${ }^{12}$ and hydrogen peroxide ${ }^{29}$ reduction or electrodeposition ${ }^{30}$, can be catalyzed, or the nanoparticle itself can be oxidised ${ }^{12}$ or reduced $^{31}$, and a corresponding current spike of typically 2 to $20 \mathrm{~ms}$ duration is observed. ${ }^{8,32}$

This way a variety of information on the impacting nanoparticles 90 can be derived. The onset potential of oxidative current spikes can be used to identify the chemical composition of an impacting 


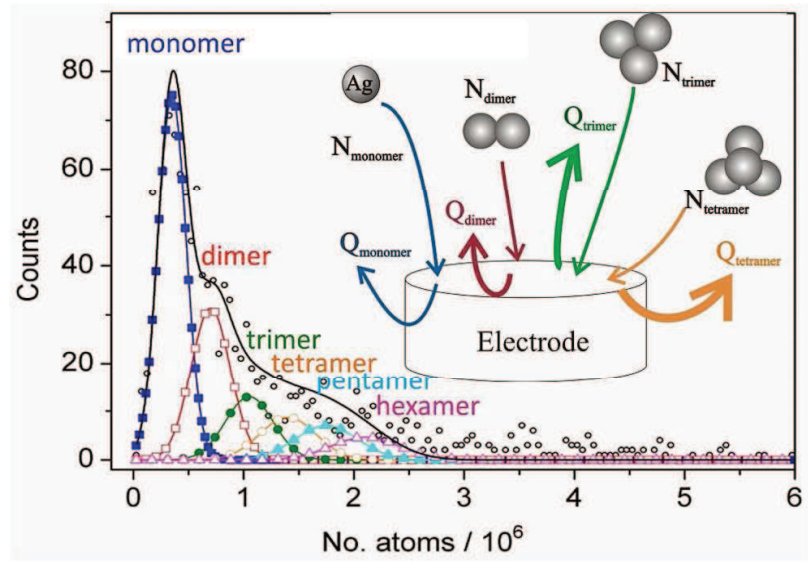

Fig. 5 Agglomeration state of silver nanoparticles (radius $=11 \mathrm{~nm}$ ) in an aqueous solution of $0.09 \mathrm{M} \mathrm{KCl}$ and $0.01 \mathrm{M} \mathrm{NaC}_{6} \mathrm{H}_{7} \mathrm{O}_{7}$, as determined by anodic particle coulometry (APC). The principle of using APC to size

5 agglomerates impacting a micro electrode is schematically summarised in the inlay. The charge per impact spike (and the number of atoms per particle) relate as 1:2:3:4:5:6 for impacting monomers, dimers, trimers,

tetramers, pentamers and hexamers, respectively; adapted from ${ }^{35}$.

10 nanoparticle. ${ }^{12,33}$ Additionally, the frequency at which spikes are detected allows for the determination of the nanoparticle concentration $^{33}$ and the charge associated with an individual spike can be used to measure the size of a single nanoparticle. ${ }^{8}$

Moreover, the agglomeration state of nanoparticles in a specific 15 liquid environment can also be studied this way. ${ }^{34,35}$ For instance, the agglomeration of silver nanoparticles in a solution containing $90 \mathrm{mM}$ potassium chloride and $10 \mathrm{mM}$ mono-sodium citrate has been studied via impact based anodic particle coulometry ${ }^{35}$. There, the anodic charge required for quantitative oxidation of a 20 particle while impacting a micro electrode is measured and related to the number of atoms in this nanoparticle, which is then related to the number of nanoparticle monomers, yielding the size of each impacting agglomerate.

The only major requirement for nanoparticle impact experiments 25 is that the electrolyte used provides sufficient electrical conductivity, allowing for a wide range of environmentally and biologically relevant solutions to be analysed with respect to their effect on silver nanoparticle agglomeration. This is highly beneficial in then understanding the afore-mentioned extreme

30 sensitivity of silver nanoparticles to their surrounding medium and will not only be a function of the composition, ionic strength and $\mathrm{pH}$ of the electrolyte, but will also strongly depend on the size and capping agent of the nanoparticle, opening up a wide field of interesting questions to address within future work

35 Despite the inherent demand for sufficient electrolyte conductivity, impact experiments are also suitable for studying individual nanoparticles when using an appropriate, nonagglomerating electrolyte. Very recently $0.1 \mathrm{M}$ tri-sodium citrate solution was found to be an electrolyte of this kind, allowing 40 citrate-capped silver nanoparticles to be studied in their monomer form. ${ }^{36}$ In this work four different samples of silver nanoparticles, each of different average size and size distribution, were sized by anodic particle coulometry during nanoparticle impact experiments in the $0.1 \mathrm{M}$ citrate solution. Additionally, SEM 45 analysis of the nanoparticles (in their non-agglomerated stock

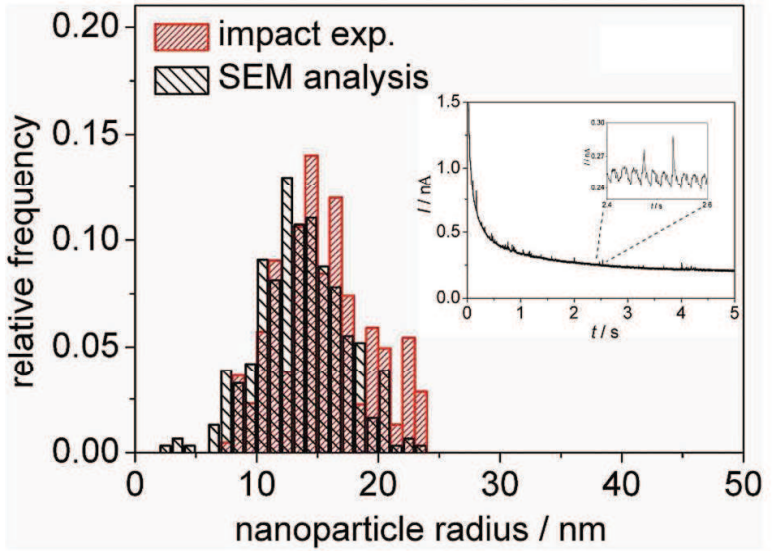

Fig. 6 Comparison of the size distributions of a sample of silver nanoparticles as determined by nanoparticle impact experiments (red columns, $r=9.2 \pm 2.2 \mathrm{~nm}$ ) in $0.1 \mathrm{M}$ tri-sodium citrate solution and SEM 50 analysis (black columns, $r=8.5 \pm 3.5 \mathrm{~nm}$ ); the inlay shows a SEM images of the analysed silver nanoparticles (adapted from ${ }^{36}$ )

suspension state) was performed using a TEM grid modified sample holder to minimise nanoparticle agglomeration upon the ${ }_{55}$ drying of the sample. Hence, sizing of the silver nanoparticles was performed via two individual routes and both showed excellent agreement in the mean size and size distribution determined for each sample, as shown in Figure 6. This clearly indicates that in contrast to the impact experiment presented 60 before (see Fig. 5), agglomeration of the citrate-capped silver nanoparticles is minimised in the presence of an electrolyte that only contains citrate, but no chloride anions.

This impact methodology is remarkably flexible owing to the wide range of experimental parameters that can be adjusted ${ }_{65}$ specifically (such as the electrolyte) to address particular analytical tasks (e.g. the investigation of agglomerates or nanoparticle monomers). In combination with its inherent sensitivity to single particle characterization, it is anticipated that this method will be more and more widely used for fundamental 70 electrochemical studies, for example addressing, quantifying and understanding possible catalytic effects of nanoparticulate silver. Simultaneously, this technique does already and will further branch towards more diverse types of nanoparticle, such as metal oxides $^{35}$ or organic nanoparticles ${ }^{37}$.

${ }_{75}$ Clearly, the limits of nanoparticle impact experiments today are determined by the signal to noise ratio of the commercially available electrochemical equipment. Since any detected current spike, originating either from a nanoparticle surface mediated reaction or from oxidation of the silver nanoparticle upon 80 impacting an electrode, directly depends on the nanoparticle size, the minimum size that can be analysed has to yield a signal that can be clearly distinguished from the background noise. To the authors' knowledge, this is currently in the range of about $5 \mathrm{~nm}$ radius, when anodic particle coulometry for silver nanoparticles ${ }_{85}$ is concerned (see Fig. 6). ${ }^{36}$ This is already as low as alternative liquid-phase sizing options, such as claimed by the producers of Nanoparticle Tracking Analysis, ${ }^{38}$ but does furthermore allow the derivation of a variety of additional electrochemical information, which is not true for optical techniques. Small nanoparticles can 90 be studied either by one of the above presented solution-based 
sticking methods or by nanoparticle mediated electrochemical impact experiments (allowing a larger charge per spike to be generated than in the case of destructive impact experiments of small nanoparticles).

\section{Conclusions}

The variety of existing electrochemical techniques to analyse nanoparticles, in particular silver nanoparticles, illustrates both the enormous demand for reliable techniques to characterise 10 nanoparticulate materials and the great potential of electrochemical approaches to satisfy this demand. The above guide seeks to support future efforts in nano-electrochemistry in order to extend its use in nanoparticle analysis and characterization. The application of the discussed methodologies 15 is not limited to metal nanoparticles, such as silver and gold ${ }^{8}$, but also studies involving metal oxide ${ }^{31}$ and organic nanoparticles ${ }^{37}$ were performed. Additionally, the combination of these electrochemical methodologies with in situ spectroscopic techniques opens up a highly interesting research field for the 20 physical chemist.

\section{Acknowledgments}

This research was supported by a Marie Curie Intra European Fellowship within the 7th European Community Framework ${ }_{25}$ Programme (KT). CBM and RGC acknowledge funding from the European Research Council under the European Union's Seventh Framework Programme (FP/2007-2013) / ERC Grant Agreement n. [320403]. HST thanks the National Research Foundation Singapore under its National Research Foundation (NRF) 30 Environment and Water Technologies (EWT) PhD Scholarship Programme and EJES thanks the Leverhulme Trust (Grant No. F/08 788/J) for financial support.

\section{${ }_{35}$ Notes and references}

Department of Chemistry, Physical \& Theoretical Chemistry Laboratory, Oxford University, South Parks Road, Oxford, OX1 3QZ, United Kingdom. Fax: +44(0) 1865 275410; Tel: +44(0) 1865 275957; E-mail: richard.compton@.chem.ox.ac.uk

Following the IUPAC definition ${ }^{22}$ the terms agglomeration and aggregation denote the reversible or irreversible adhesion of particles, respectively. The analytical techniques referred to in this perspective detect both simultaneously. Thus, the term agglomeration will be used to refer to both types of particle association for mutual convenience.

2 Most applications and experimental studies today employ electrostatically stabilized citrate-capped silver nanoparticles. The repulsive force between these negatively charged single nanoparticles is shielded and hence agglomeration is favoured in electrolytes of high ionic strength, as explained by the Derjaguin-Landau-Verwey-Overbeek (DLVO) theory ${ }^{26,27}$.

${ }^{3}$ For instance, the stripping charge expected for the sticking experiments described below is $0.3 \mathrm{nC}$ (see Fig. 2). Electrodeposition of the corresponding amount of silver over the duration of the sticking
}

experiment $(15 \mathrm{~min})$ requires a $\mathrm{Ag}^{+}$contamination of only $3 \times 10^{-8} \mathrm{M}$ (assuming diffusion limited deposition and $D\left(\mathrm{Ag}^{+}\right)=1.648 \times 10^{-5} \mathrm{~cm}^{2} \mathrm{~s}^{-1}$ $\left.{ }^{28}\right)$.

${ }^{4}$ The $\mathrm{Ag} / \mathrm{Ag}\left(\mathrm{NO}_{3}\right)$ reference electrode was fabricated by immersing a $\mathrm{Ag}$ wire in a solution containing $0.01 \mathrm{M} \mathrm{Ag}\left(\mathrm{NO}_{3}\right)$ and $0.09 \mathrm{M} \mathrm{Na}\left(\mathrm{NO}_{3}\right)$; a platinum mesh served as the counter electrode in this study.

Platinum mesh and carbon counter electrodes were studied this way, both yielding the same trend. A solely solution-phase redox reaction related aging effect of the electrolyte was excluded, as the same increase in stripping charge was observed when either leaving only a few minutes in between experiments or when leaving the electrolyte over night. Note that this does not proof that chemical ageing (for instance Ag nanoparticle dissolution) can be excluded, but only that the electrochemical $\mathrm{Ag}^{+}$ formation exceeds the chemical one in the presented experiment.

\section{References}

1. R. Kessler, Environ. Health Perspect., 2011, 119, A120-A125.

2. S. Chernousova and M. Epple, Angew. Chem. Int. Ed., 2013, 52, 1636-1653.

3. J.-Y. Bottero, M. Auffan, J. Rose, C. Mouneyrac, C. Botta, J. Labille, A. Masion, A. Thill, and C. Chaneac, Comptes Rendus Geosci., 2011, 343, 168-176.

4. R. Behra, L. Sigg, M. J. D. Clift, F. Herzog, M. Minghetti, B. Johnston, A. Petri-Fink, and B. Rothen-Rutishauser, J. R. Soc. Interface, 2013, 10.

5. A. G. Howard, J. Environ. Monit. JEM, 2010, 12, 135-142.

6. J. Liu and R. H. Hurt, Environ. Sci. Technol., 2010, 44, 2169-2175.

7. X. Li, J. J. Lenhart, and H. W. Walker, Langmuir, 2010, 26, 1669016698.

8. N. V. Rees, Y.-G. Zhou, and R. G. Compton, RSC Adv., 2012, 2, 379.

9. E. J. E. Stuart, K. Tschulik, D. Omanović, J. T. Cullen, K. Jurkschat, A. Crossley, and R. G. Compton, Nanotechnology, 2013, 24, 444002

10. K. Tschulik, R. G. Palgrave, C. Batchelor-McAuley, and R. G. Compton, Nanotechnology, 2013, 24, 295502.

11. W. Cheng, E. J. E. Stuart, K. Tschulik, J. T. Cullen, and R. G. Compton, 2013, in press.

12. Y.-G. Zhou, N. V. Rees, and R. G. Compton, Angew. Chem. Int. Ed., 2011, 50, 4219-4221.

13. E. J. E. Stuart, N. V. Rees, J. T. Cullen, and R. G. Compton, Nanoscale, 2012, 5, 174-177.

14. C. N. R. Rao, G. U. Kulkarni, P. J. Thomas, and P. P. Edwards, Chem. Soc. Rev., 2000, 29, 27-35.

15. R. W. Murray, Chem. Rev., 2008, 108, 2688-2720.

16. C. Bai and M. Liu, Angew. Chem. Int. Ed., 2013, n/a-n/a.

17. C. Levard, E. M. Hotze, G. V. Lowry, and G. E. Brown, Environ. Sci. Technol., 2012, 46, 6900-6914.

18. C. Batchelor-McAuley, C. C. M. Neumann, K. Tschulik, and R. G. Compton, 2013 submitted

19. H. S. Toh, C. Batchelor-McAuley, K. Tschulik, and R. G. Compton, The Analyst, 2013, 138, 4292.

20. H. S. Toh, C. Batchelor-McAuley, K. Tschulik, M. Uhlemann, A. Crossley, and R. G. Compton, Nanoscale, 2013, 5, 4884-4893.

21. T. J. Davies, B. A. Brookes, and R. G. Compton, J. Electroanal. Chem., 2004, 566, 193-216.

22. IUPAC Compendium of Chemical Terminology - the Gold Book, International Union of Pure and Applied Chemistry, 2012, vol. 2.3.2.

23. Y.-G. Zhou, N. V. Rees, and R. G. Compton, Chem. Phys. Lett., 2011, 514, 291-293.

24. C. C. M. Neumann, C. Batchelor-McAuley, K. Tschulik, H. S. Toh, P. Shumbula, J. Pillay, R. Tshikhudo, and R. G. Compton, ChemElectroChem, 2013, in press, doi: 10.1002/celc.201300062.

25. W. J. Plieth, J. Phys. Chem., 1982, 86, 3166-3170.

26. B. Derjaguin and L. Landau, Prog. Surf. Sci., 1993, 43, 30-59.

27. E. J. W. Verwey and J. T. G. Overbeek, Theory of the Stability of Lyophobic Colloids, Elsevier, 1948.

28. D. R. Lide, CRC handbook of chemistry and physics: a readyreference book of chemical and physical data: 2008-2009, Taylor \& Francis Group, 89th edn., 2008. 
29. E. J. E. Stuart, N. V. Rees, and R. G. Compton, Chem. Phys. Lett., 2012, 531, 94-97.

30. Y.-G. Zhou, N. V. Rees, and R. G. Compton, Chem. Phys. Lett., 2011, 511, 183-186.

31. K. Tschulik, B. Haddou, D. Omanović, N. V. Rees, and R. G. Compton, Nano Res., 1-6, in press doi:10.1007/s12274-013-0361-3.

32. D. Hellberg, F. Scholz, F. Schubert, M. Lovrić, D. Omanović, V. A. Hernández, and R. Thede, J. Phys. Chem. B, 2005, 109, 1471514726.

33. E. J. E. Stuart, Y.-G. Zhou, N. V. Rees, and R. G. Compton, RSC Adv., 2012, 2, 6879-6884.

34. N. V. Rees, Y.-G. Zhou, and R. G. Compton, ChemPhysChem, 2011, 12, 1645-1647.

35. J. Ellison, K. Tschulik, E. J. E. Stuart, K. Jurkschat, D. Omanović, M. Uhlemann, A. Crossley, and R. G. Compton, ChemistryOpen, 2013, $2,69-75$.

36. J. C. Lees, J. Ellison, C. Batchelor-McAuley, K. Tschulik, C. Damm, and D. Omanović, ChemPhysChem, 2013, in press, doi:10.1002/cphc. 201300796 .

37. W. Cheng, X.-F. Zhou, and R. G. Compton, Angew Chem Int Ed, 2013, in press, doi:10.1002/anie.201307653.

38. http://www.nanosight.com/publications/technical-notes, NanoSight, Amesbury, UK, 2009. 